\title{
A Meta-Analysis on the Application of SNS in Education for SMART Learning: Focusing on Korean Case
}

\author{
Soon-Hwa Kim ${ }^{1}$, Ki-Sang Song ${ }^{2}$ \\ ${ }^{1}$ Department of Computer Education for the Gifted, Korea National University of Education, Chung-won, Korea \\ ${ }^{2}$ Department of Computer Education, Korea National University of Education, Chung-won, Korea \\ Email: soona6570@gmail.com, kssong@knue.ac.kr \\ Received 2012
}

\begin{abstract}
As the Smart Phone and iPad type devices widely spread all over the world, the Social Network Service (SNS) has become prevalent. The SNS deeply penetrates into personal lives, it also affects learning and education. To evaluate the SNS effects on education, we have analyzed 153 articles from 2009 to 2012 published in Korea to know the current status of the application of the SNS on education in Korea. From this meta-analysis review, we found that the Wiki service is the most widely researched SNS service applicable to education in Korea, and information technology subject is the hottest discipline when considering SNS for education. Also, college level education is the most well prepared school type to use SNS for education. This review results suggest that the most appropriate level of applying smart education education using smart phone or iPad type devices - is college level or above instead of K-12 education.
\end{abstract}

Keywords: Social Network Service; SNS; Education

\section{Introduction}

Social Network Service (hereinafter SNS) is an online service, platform, or site that focuses on building and reflecting of social networks or social relations among people (Wikipedia, 2012). Most of SNS are web-based services to interact over the Internet or a mobile device. According to the survey of Korea Internet Security Agency 61.3\% of Internet users are using SNS (Internet, Security Agency, 2007). The actual membership of SNS in Korea dramatically increased with the spread of smart mobile devices. According to the survey from each SNS company, the number people in Korea who have a Twitter membership is 3.5 million, a Facebook membership is 4 million, and Me2day is 5.7 million. More than 12.7 million people have SNS memberships. With the wide spread of the Internet and smart mobile devices, the number of SNS users have been surprisingly increasing in Korea. There are many kinds of SNS, widely used such as wiki, Facebook, Twitter, Me2day, blog, Internet café and so on in Korea. These services are ranging from individual communication to group communication and from individual content production to group content production.

As the smart devices are rapidly adopted in every field of society, the Korean Ministry of Education, Science and Technology set the master plan for SMART education (Ministry of Education Science and Technology, 2011). According to the Ministry presentation, SMART education means self-directed(S), motivated (M), adaptive(A), resource free(R), technology embedded(T) education. It focuses on activating online education with digital contents using smart device. It also includes an enhancement plan using SNS with various smart devices. Nowadays, SMART learning is the key issue in Korean educational field.

Many of Korean researchers in the education and technological fields are actively researching SMART learning with SNS from 2010 till now. K. S. No, etc(2011) established the definitions of
SMART learning through studying previous works on SMART education. SMART learning is a learner initiated learning which has various materials for learning and supports learner-teacher interaction (Noh, Ju, \& Jung, 2011) J. H. Kang(2011) suggested that SNS is an effective tool for enhancing student initiated learning especially the youth. According to her research, the Korean government has to support SNS use by revision of law and social system. In addition, people have to have a changed perception of using SNS (Kang, 2011).

SMART is not a notion that suddenly appeared. It is an extension of e-learning and u-learning. In order to implement the Korean government's master plan for SMART learning, a close examination of SNS for education research has to be preceded. Also J. H. Kang(2011) pointed out difficulties in researching SNS because of a lacking in advanced research. Several characteristics are summarized within the whole body of research following.

\section{The Basic Framework of Research}

To understand the main concern of educators and education researchers in SNS based education, this paper analyzed papers published last four years (from 2009 to 2012) including journals, master's and doctor's thesis in Korea.

\section{The Main Framework of SNS}

General classification has to be preceded before analysis begins, to get a better understand of SNS since SNS's are emerging as the technological innovation. The SNS can be classified with the purpose, to make instant messages or produce contents. In addition to that, user based standard can be used to make classifications, such as individual communication as in oneto-one or group communication. According to these standards, we can suggest a basic framework to analyze the social media applications to education as shown in Figure 1. 
As we can see in the Figure 1, Kakaotalk ${ }^{1}$, Nateon ${ }^{2}$, and MSN are the individual oriented SNS which mainly focus on exchange of instant messaging. Also these SNS have functions to exchange instant messaging among group members. Internet café and clubs, on the other hand, are the group oriented SNS, which mainly focus on making communication with people who have the same interests. Next type of SNS such as Wikipedia, Springnote, Googledocs, are group oriented, collaborative SNS to produce contents. Blog, Tistory ${ }^{3}$, and Cyworld are individual oriented SNS which are mainly used for producing contents. Also they have somewhat closed characteristics compared to group oriented SNS like Wikipedia. The final category is one of the most emerging classes, such as Facebook, Twitter, and Me2day in Korea. This type of SNS is individual oriented, which can communicate or share contents and message with a person or groups. The user can take advantage of its characteristics for their own purpose.

Based on the above framework of SNS, this paper selected commonly used SNS as research objects. In this paper comprehensive researches have searched related to the application of SNS in education including Facebook, Twitter, Wiki, Blog, Me2day and other specific SNS.

\section{Literature Source}

Research Information Sharing Service (RISS) which is supported by Korea Education Research Information Service (KERIS) has been used to find articles about SNS based education. RISS is widely used and it has comprehensive information about current education in Korea. Keyword search is the effective method to select related researches about research objects.

From 2009 to 2012, hundreds of articles with "social network service" and the specific SNS for keywords were retrieved from RISS (www. Riss4u.kr). The Articles that have no relation with education were excluded. Finally, 153 articles were selected which closely related to the research subject, the application of SNS in education.

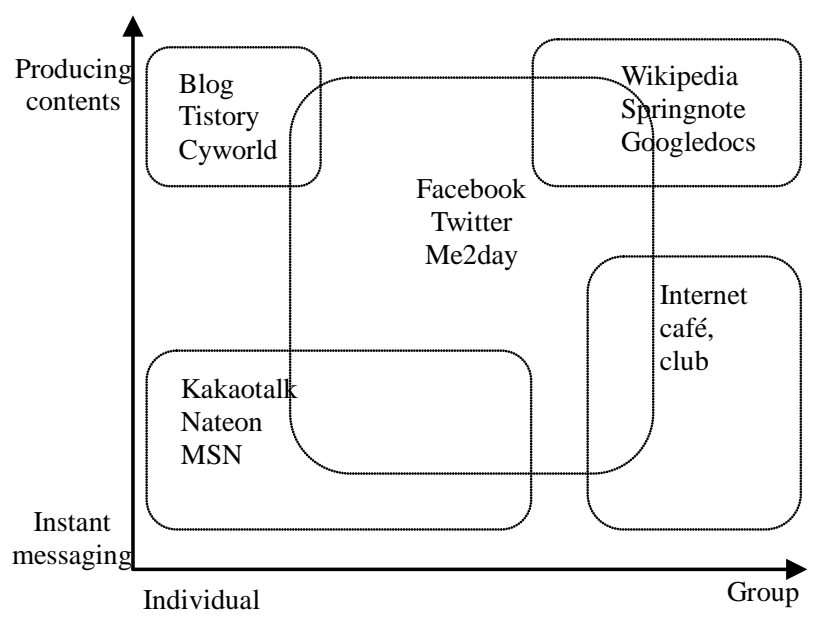

Figure 1.

Basic framework in analyzing SNS research in education.

${ }^{1}$ Kakaotalk is a free short messaging service provided Kakaotalk.com. It can deliver not only text but also multimedia data, and can be used as smart mobile device application.

${ }^{2}$ Nateon is a chatting program available for smart device and PC.

${ }^{3}$ Tistory is a blog site operated in Korea.

\section{Analysis on the Application of SNS for Education}

\section{The Overall Situation of SNS Usage in Education}

There are 153 categorized articles with the type of the SNS that is used for research (see Figure 2).

In the distribution graph of the type of SNS, 38\% articles are about researches on Wiki based education. This can be explained by ICT advancement in Korea. With the well organized computer facilities in public school and students' owning PCs, students and teachers can access the Internet conveniently. Also Wiki may support group collaborative work, and therefore educators and researchers have more concerns in cooperative tasks with Wiki compared to other social media. The next highly researched area is about Twitter on education, 18\% of the analyzed papers were published on this subject. Wide spread smart mobile devices stimulate the research on Twitter based education, especially for online. On the other hand, only $9 \%$ of articles are about Facebook. It is not easy to explain this phenomena, but it may be due to the recent introduced social media form in Korea compared to other SNS types. The main purpose of Facebook usage is usually building relationships with friends, thus educational researchers may have little concern about Facebook and education yet.

Also, the multimedia delivered by the service may affect to the SNS application to education research. For example, Nateon and Kakaotalk mainly focus on real-time chatting are not easy to use in education. As the Figure 2 shows, researches on the applications are very few, and despite of the popularity of Nateon and Kakaotalk in Korea, little research has been done yet. Presumably the lack of usefulness in education is the main cause of the result.

We also analyzed the yearly distribution of 153 articles through 4 years from 2009 to 2012 (see Figure 3). As we can see above Figure 3, SNS for education has been widely used since 2010. Before 2010, almost of articles are about wiki or blog. 24 articles were retrieved in 2009, the frequency of research has dramatically increased since 2010. 43\% of articles were distributed in 2011. The total number of articles about SNS based learning is still increasing taking in consideration the retrieved date (April 2012). The rising number of Facebook is quite notable.

In conclusion, SNS have been widely used and became a hot issue in education since 2010 within Korea. This is closely related to the spreads of smart mobile devices from students to teachers, thus the familiarity of SNS has been increased among Korean people.

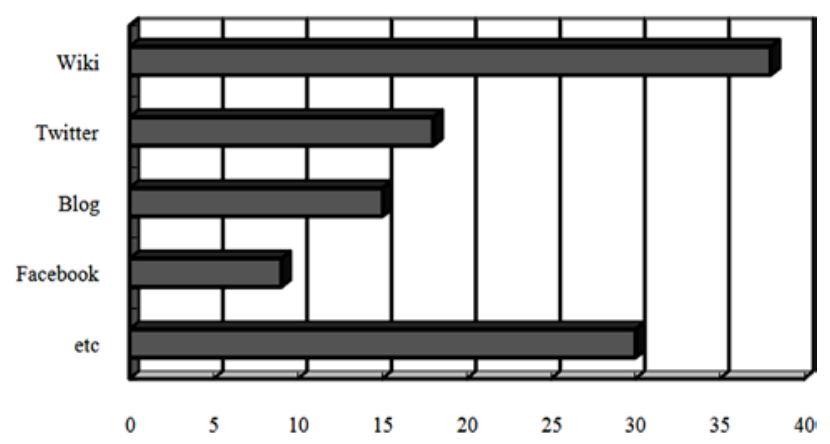

Figure 2.

The type of SNS distribution (\%). 


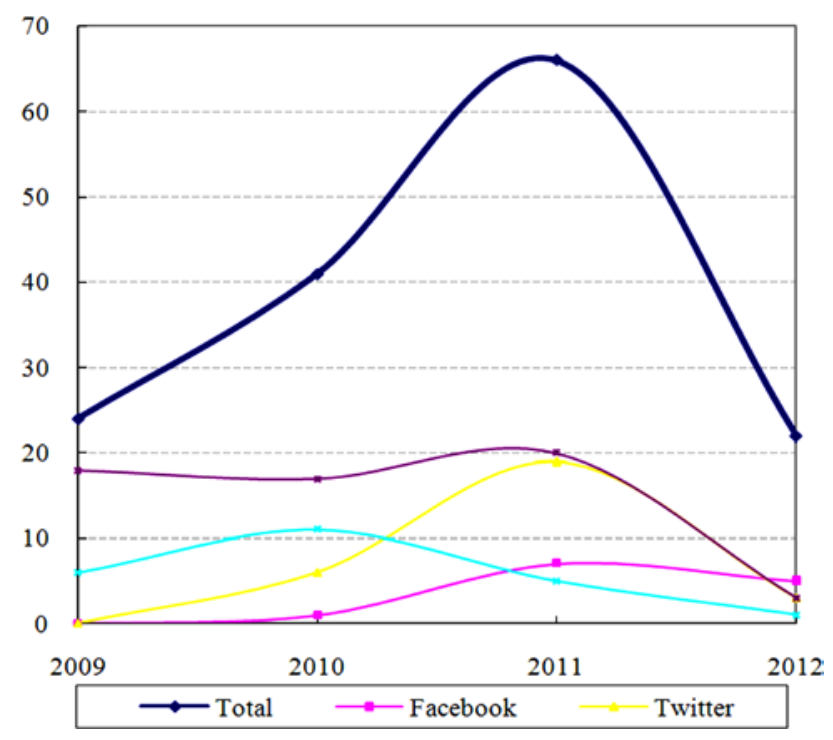

Figure 3.

Annual distribution of article.

To figure out which level of school types are more applicable to SNS and education, we analyzed papers according to the school level; primary, secondary, college level (see Figure 4).

As the Figure 4 shows, 31\% of the articles research college level students. The ages 20-30s are the most widely SNS using group in Korea which closely links with the use of smart mobile device. Next, $19 \%$ of the articles are about secondary school students. However, compared to college and secondary school level, Primary school has the smallest amount of article researched.

This may due to the difficulty of treating smart mobile device for the primary school students. In addition, there are few primary school students have smart mobile device.

To determine researchers study the application of social media within education, we investigated the research method applied to each article; empirical research and non-empirical research.

As the Table 1 shows, the empirical research method occupied $70 \%$ of the papers. The ratio comparing to non-empirical research seems relatively high. However, considering the importance of the empirical method and the non-empirical method, further research is required using the non-empirical method. As you can see in Table 1, the empirical researches are using experimental method more than any other. Case study method is relatively rare in SNS for educational research.

\section{Domain of the SNS Application in Education}

1) Subject related articles

The distribution of subject that SNS used is shown Figure 5. As shown Figure 5, the distribution leans too much towards IT and language. $44 \%$ of the articles are about IT, such as teaching programming, cyber ethics and so on. This is due to the main tool of teaching being an IT device. Because collaborative work is effective when teaching IT related subjects, SNS based education in IT subjects has widely been researched.

$38 \%$ of the articles are related to language education, such as foreign language learning, especially, using Twitters and Facebook. It is may be due to the main characteristics of Twitters and Facebook being more appropriate for teaching language with short sentences.

Since both Twitter and Facebook are easy to create individual contents and delivering news just adding a few sentences along with communication with short sentences, thus exposure time of language can be increased through students using this SNS. Only a small proportion of articles are related to other subjects, so further studies are needed to find an adequate way of applying of SNS.

2) The remainder but in domain of education

From this meta-analysis, we found that there exist articles not directly related to any subject but focus on teaching and learning. These articles can be classified as collaborative learning, social relationship with students, learning strategy and so on.

As you can see in Figure 6, 49\% of the articles are related to learning, latent learning among people to people using SNS, smart education, learning satisfaction level and collaborative learning are the specific topic of this field. It reflects the emerging concern about SNS for education among Koreans. In fact, articles in this domain were intensively distributed in 2011 and 2012 . 32\% of the articles are about social relationships, considering online relation affects to offline relation, interaction case study, building a relationship through SNS is the main

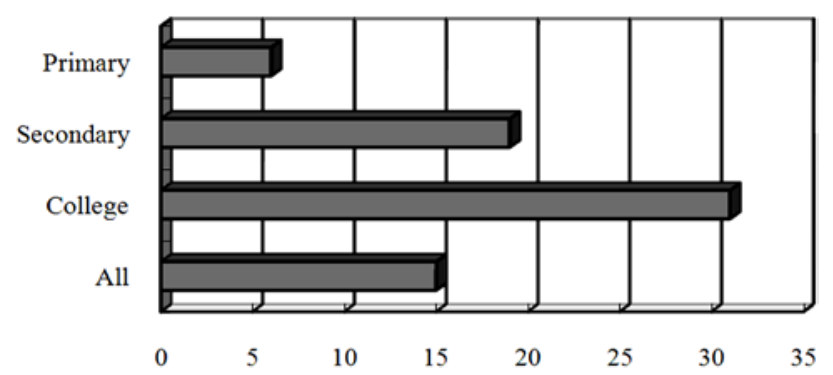

Figure 4.

Distribution of school level.

Table 1.

Research method distribution.

\begin{tabular}{ccccc}
\hline \multicolumn{5}{c}{ Empirical method } \\
\hline Experiment & Survey & Literature review & Action research & Case study \\
\hline $36 \%$ & $19 \%$ & $19 \%$ & $20 \%$ & $6 \%$ \\
\multicolumn{4}{c}{ Non-empirical method } \\
\multicolumn{3}{c}{$30 \%$} \\
\hline
\end{tabular}

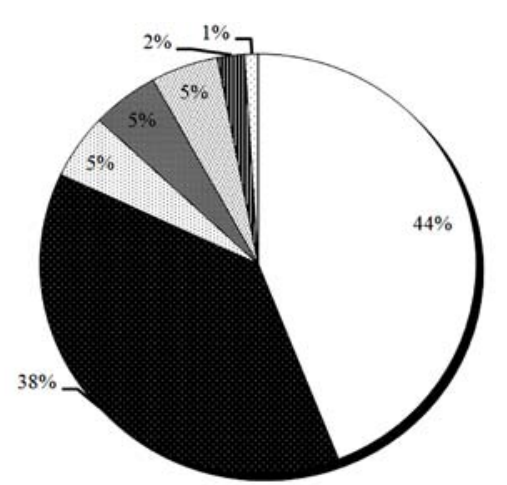

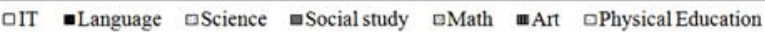

Figure 5.

Distribution of subject related SNS articles. 


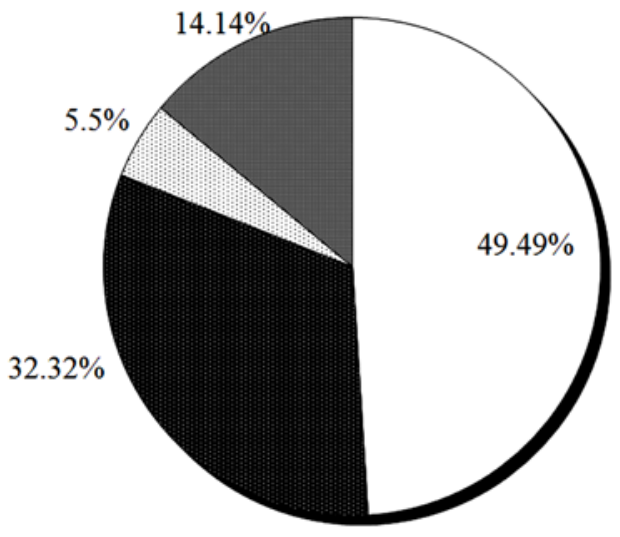

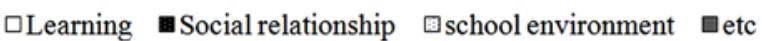

Figure 6.

Article distribution not related to subject.

topic of this field. Through the fact that online friendship can affect offline friendship (Kim, Choi, 2012), we can realize that collaborative learning can be effective with the application of SNS in education.

\section{Conclusion and Suggestions}

\section{Summary of Characteristics}

1) Rising types of SNS in Korean education

Many teachers and researchers have more concern about SNS's application in education. As you can see Figure 2, the aspect of each SNS article's annual distribution change has a different pattern. The article related with Twitter and Facebook is increasing, but Wiki and Blog's are decreasing. It reflects the effect of Twitter and Facebook on education. Korean government's master plan, SMART learning can be easily accepted to the subjects who are in the educational field such as teachers and students.

2) Biased article distribution in subject and school level

There exists uneven pattern in research of SNS for education. For example, subjects are seriously biased to IT and language education. Little research has been done in the other subjects such as math, science, art, music, and so on. Definitely, SNS can be used to teach another subject. Also, there exists little research on the application of SNS at the primary school level (see Figure 4). In order to accomplish the master plan for SMART learning, primary schools have a concern for researchers. Effective ways of applying SNS to primary school students has to be researched and developed.

3) Lack of microscopic view reflecting each characteristics of SNS

Each SNS has its own characteristics. Articles seldom consider its detailed characteristics. In other words, researchers are taking a macroscopic view when applying SNS on education. Therefore, thorough analysis needs to be preceded before applying SNS on education.

\section{Suggestions for Further Research}

1) Taking microscopic view when applying SNS on education Continuously improve teacher and researcher's digital literacy so that they can find out the effective way of applying SNS. If they have detailed knowledge of the characteristics of each SNS, they can take advantage of SNS and more conveniently teach students. Furthermore, an adequate education model can be developed when teaching students with SNS.

2) Collaborative learning model using SNS

Online relationships can affect offline relationships as well (Kim, Choi, 2012). Considering the positive relationship among group members affects team efficacy (Johnson, Johnson, 1992). Twitter or Facebook based collaborative learning model can be an interesting subject for educators.

3) Balancing the research level and subject

Primary education is the core of education. Though it is difficult, because primary students are too young, finding an effective way to apply SNS can be a critical problem for the educators and researchers. Also, finding effective ways to apply SNS to various subjects can be a task for researchers.

\section{REFERENCES}

"Wikipedia”, URL (last checked 23 April, 2012) http://en.wikipedia.org/wiki/Social_network_service, Internet Security Agency (2009). Survey of SNS membership status of the Internet users.

T.J. Sung (2007). The Methodology of Research. Hakjisa..

M.J. Kim and Y.J. Choi (2012). The Role of Twitter Online and Offline Relationship Formation, Korean Journal of Broadcasting, 26(1), 43-82.

Ministry of Education, Science and Technology (2011). SMART Education Strategies.

D.W. Johnson and R.T. Johnson (1992). Positive Interdependence: Key to Effective Cooperation. Interaction in cooperative groups, New York: Cambridge University Press.

K. S. Noh, S. H. Ju, J. T. Jung (2011). An exploratory study on concept and realization conditions of smart learning, The journal of digital policy \& management, 9(2), 79-88.

J. H. Kang(2011) A study on utilizing SNS to vitalize smart learning. The journal of digital policy \& management, 9(5), 265-274. 